\title{
Importancia de la prueba de calprotectina fecal en la enfermedad inflamatoria intestinal
}

\section{Importance of the fecal calprotectin test in inflammatory bowel disease}

Brenda Maldonado-Arriaga*

Laboratorio de Metabolismo Experimental e Investigación Clínica, División de Investigación Clínica, Centro Médico Nacional 20 de Noviembre, Instituto de Seguridad y Servicios Sociales de los Trabajadores del Estado, Ciudad de México, México

\begin{abstract}
Resumen
La enfermedad inflamatoria intestinal (EII) es un problema sanitario mundial con una incidencia en aumento continuo. Se caracteriza por una inflamación intestinal crónica e incluye dos condiciones clínicas: la enfermedad de Crohn y la colitis ulcerativa. Hasta hace poco, el diagnóstico se basaba principalmente en criterios clínicos y endoscópicos, por lo que ha existido la necesidad de utilizar una prueba no invasiva para el diagnóstico, el pronóstico y el seguimiento de la Ell. Hasta la fecha, la calprotectina fecal (CF) es la prueba más estudiada y la que mejores resultados ha mostrado. La calprotectina es una proteína de unión al zinc y al calcio de $36 \mathrm{kDa}$ que es expresada principalmente por los neutrófilos. La concentración de CF aumenta debido a la agregación de neutrófilos en la mucosa del intestino inflamado; por tanto, es útil como marcador no invasivo de la inflamación en la Ell. La prueba de CF es no invasiva, estable, simple, fácil de realizar, rápida y reproducible. La determinación de la CF contribuye a la evaluación del grado de actividad de la enfermedad, al seguimiento de la respuesta terapéutica y a predecir la recaída en la Ell. En esta revisión se aborda la importancia de la CF en la fase activa y en la fase de remisión de la Ell.
\end{abstract}

Palabras clave: Enfermedad inflamatoria intestinal. Calprotectina fecal. Fase activa. Fase de remisión.

\section{Abstract}

Inflammatory bowel disease (IBD) is a global health problem with a continuously increasing incidence. It is characterized by chronic intestinal inflammation, made up of two clinical entities: Crohn's disease and ulcerative colitis. Until recently, the diagnosis consisted mainly of clinical and endoscopic criteria. Therefore, there has been a need to use a non-invasive test for the diagnosis, prognosis and follow-up of IBD. To date, fecal calprotectin (CF) is the most studied test and the one that provides the best results. Calprotectin is a $36 \mathrm{kDa}$ zinc and calcium binding protein expressed primarily by neutrophils. The concentration of CF increases due to the aggregation of neutrophils in the mucosa of the inflamed intestine; therefore, it is useful as a non-invasive marker of inflammation in IBD. The CF test is non-invasive, stable, simple, easy to perform, fast, and reproducible. Determining the level of CF contributes to the evaluation of the degree of disease activity, the monitoring of the therapeutic response, and the prediction of relapse in IBD. Therefore, this review addresses the importance of CF in the active phase and in the remission phase of IBD.

Key words: Inflammatory bowel disease. Fecal calprotectin. Active phase. Remission phase.

Correspondencia:

*Brenda Maldonado-Arriaga

E-mail: adnerb_rock@ @otmail.com
Disponible en internet: 26-05-2021

Fecha de aceptación: 06-02-2021

DOI: 10.24875/IMIDS.M21000001
Rev Mex Enferm Inflam Inmunomed. 2021;95(1):1-5

www.IMIDsMexico.com

2696-6867 / @ 2021 Permanyer. Éste es un artículo open access bajo la licencia CC BY-NC-ND (http://creativecommons.org/licenses/by-nc-nd/4.0/). 


\section{Introducción}

La enfermedad inflamatoria intestinal (EII) se define como un conjunto de trastornos inflamatorios crónicos, de causa desconocida; sin embargo, la hipótesis dominante sugiere que el desarrollo de la inflamación es por una respuesta inmunitaria anómala hacia la microbiota intestinal dentro de un hospedero genéticamente susceptible. La Ell incluye dos afecciones diferentes desde el punto de vista tanto clínico como morfológico: la colitis ulcerativa (CU) y la enfermedad de Crohn (EC); y se caracteriza por episodios de exacerbación y remisión $n^{1,2}$.

El diagnóstico de la Ell es multidisciplinario y se logra mediante hallazgos clínicos, de laboratorio, endoscópi$\cos$, histológicos y radiológicos, siendo el método de referencia para el diagnóstico y el seguimiento la evaluación endoscópica ${ }^{3}$. Por otro lado, los análisis de sangre convencionales, como la determinación de la concentración de la hemoglobina, la proteína $\mathrm{C}$ reactiva, la velocidad de sedimentación de eritrocitos, las plaquetas, los leucocitos en sangre y la albúmina, aunque son útiles en la práctica clínica para la Ell, expresan respuestas sistémicas del paciente, por lo que son pruebas insuficientes para detectar la inflamación intestinal ${ }^{4}$.

En los últimos años, la colonoscopia se ha considerado la modalidad diagnóstica más precisa y la prueba de referencia para cuantificar la actividad en la EII. Sin embargo, a pesar de su utilidad, la colonoscopia tiene algunas desventajas, ya que es costosa, incómoda para el paciente y se relaciona con algunos riesgos y complicaciones durante el procedimiento ${ }^{5,6}$. Por 10 tanto, una prueba de laboratorio precisa, relativamente simple y fácilmente disponible que refleje la inflamación de la mucosa intestinal sería beneficiosa para los pacientes con Ell. Por ello, la búsqueda de un biomarcador simple, de bajo costo, no invasivo y confiable de la inflamación intestinal se ha incrementado en los últimos años. La utilidad clínica de la calprotectina fecal (CF) como biomarcador no invasivo de la inflamación en la Ell se ha evaluado en los últimos 15 años, y en la actualidad se incorpora como prueba sistemática para el diagnóstico y el monitoreo de la Ell en los laboratorios clínicos, para lo que se dispone de varias pruebas comerciales ${ }^{7,8}$. Adicionalmente, se ha adoptado como un componente estándar de las prácticas de detección y seguimiento de la Ell.

\section{Calprotectina fecal}

La calprotectina es una proteína de unión al zinc y al calcio formada por un complejo heteromérico de dos subunidades: S100A8 y S100A9. El estado de conformación y oligomerización de S100A8/A9 está impulsado por sus propiedades de unión al calcio y al zinc, lo que conduce a la formación del heterooligómero fisiológicamente activo ${ }^{9-11}$. Se deriva principalmente de los neutrófilos y los monocitos humanos, y representa alrededor del $60 \%$ de las proteínas de citosol solubles en los granulocitos de neutrófilos humanos. En consecuencia, en la Ell, la cantidad de CF es proporcional al número de neutrófilos que migran desde la pared del intestino inflamado a la mucosa ${ }^{12-14}$ (Fig. 1).

La calprotectina se conocía anteriormente como MRP8/MRP14, antígeno asociado a la fibrosis quística, calgranulina y $\$ 100$, y se ha considerado clásicamente una proteína de defensa a nivel de la superficie epitelial debido a su actividad antimicrobiana que priva a los microorganismos de los metales de transición; específicamente, implica un secuestro de $\mathrm{Zn}^{2+}$ que resulta en el deterioro de las enzimas dependientes del zinc y la inhibición del crecimiento bacteriano ${ }^{16,17}$. Se ha demostrado que tiene actividad antimicrobiana contra Escherichia coli, Klebsiella sp. y Staphylococcus aureus, así como actividad fungistática, y se ha asociado con efectos antiproliferativos e inmunomoduladores ${ }^{18}$.

La calprotectina puede detectarse y medirse en varias muestras biológicas, como plasma, orina, saliva, líquido sinovial y biopsias de colon. Su concentración varía en proporción a la gravedad de cualquier inflamación existente; sin embargo, las determinaciones más extensas se realizan en materia fecal ${ }^{19}$. La concentración de calprotectina en la materia fecal en un estado patológico es aproximadamente seis veces más alta que la del plasma normal, y de ahí el concepto de que la medición de la CF presenta unas altas sensibilidad y especificidad en la inflamación del tracto gastrointestinal ${ }^{20}$. En el campo de la gastroenterología, la CF ha recibido mucha atención debido a su aumento en las afecciones gastrointestinales orgánicas, principalmente en la Ell. El uso clínico de la CF en la Ell se resume en la tabla 1.

\section{Medición de la calprotectina fecal}

El método utilizado con mayor frecuencia es el ELISA (Enzyme-Linked ImmunoSorbent Assay), el cual se basa en el uso de anticuerpos monoclonales o policlonales dirigidos a la molécula de calprotectina dimérica $^{22,23}$. La CF es resistente a la proteólisis intestinal, y esta característica hace que sea estable durante un par de días a temperatura ambiente, por lo que la recolección de las muestras de heces podría hacerse en 


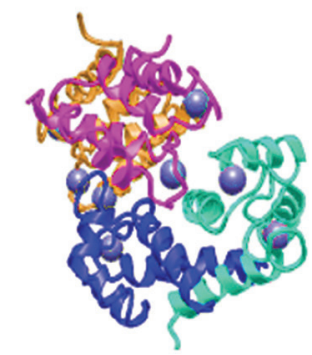

Calprotectina

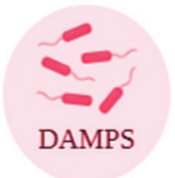

Actividad antimicrobiana
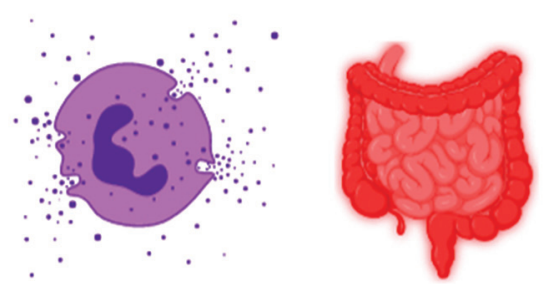

Neutrófilo
Enfermedad inflamatoria intestinal

Figura 1. Generalidades de la proteína calprotectina, una proteína de $36 \mathrm{kDa}$ perteneciente a la familia S100. Esta proteína se deriva predominantemente de los neutrófilos y representa aproximadamente el $60 \%$ de las proteínas citosólicas en los neutrófilos. La calprotectina tiene un efecto antimicrobiano directo; su mecanismo está relacionado con la capacidad de privar a los microorganismos de metales de transición. Adicionalmente, la calprotectina es parte de la respuesta inmunitaria innata, actuando como una molécula endógena asociada al patrón molecular asociado a daño (DAMP) a través de la activación del receptor tipo Toll 4. La calprotectina se ha descrito como un biomarcador que mide la inflamación en el tracto gastrointestinal en la enfermedad inflamatoria intestinal ${ }^{11,15}$.

Tabla 1. Uso clínico de la calprotectina fecal en el manejo de la enfermedad inflamatoria intestinal ${ }^{21}$

\begin{tabular}{l|l}
$\begin{array}{l}\text { Pacientes sin diagnóstico } \\
\text { previo de EII }\end{array}$ & $\begin{array}{l}\text { Detección de Ell en pacientes } \\
\text { con síntomas gastrointestinales }\end{array}$ \\
$\begin{array}{l}\text { Pacientes con diagnóstico de } \\
\text { EII }\end{array}$ & $\begin{array}{l}\text { Seguimiento de la curación } \\
\text { de la mucosa en la EC y la CU } \\
\text { Seguimiento de la recurrencia } \\
\text { de la enfermedad después de } \\
\text { la resección intestinal en la EC } \\
\text { Predicción de recaída clínica } \\
\text { en la EC y la CU }\end{array}$ \\
\hline
\end{tabular}

CU: colitis ulcerativa; EC: enfermedad de Crohn; Ell: enfermedad inflamatoria intestinal

casa y después enviarlas al laboratorio. Antes de la medición en el laboratorio, las muestras pueden ser almacenadas a $2-8^{\circ} \mathrm{C}$ durante 6 días y a $-20^{\circ} \mathrm{C}$ hasta por 4 meses $^{24}$. Se ha descrito, tanto en la literatura como en los estuches comerciales, un valor de corte de $50 \mu \mathrm{g} / \mathrm{g}$ para adultos y niños mayores de 4 años para diferenciar la Ell de otras formas de inflamación, como el síndrome de colon irritable, con una especificidad del $60 \%$ y una sensibilidad del $92 \%$, evitando así procedimientos invasivos ${ }^{25}$.

\section{La calprotectina fecal en la fase activa de la enfermedad inflamatoria intestinal}

La CF se utiliza en atención primaria y secundaria en pacientes con síntomas abdominales para ayudar a diferenciar a aquellos con Ell de aquellos otros con síndrome de intestino irritable 26,27 . El sangrado gastrointestinal es una fuente potencial de un aumento significativo de la CF; aunque está presente en la sangre, la CF entra en la luz intestinal como parte de un proceso inflamatorio y se ha calculado que requeriría una pérdida de sangre de más de $300 \mathrm{ml} /$ día en un proceso de pancolitis o un cáncer colorrectal para producir una concentración de CF de aproximadamente $150 \mathrm{mg} / \mathrm{g}$.

La CF se ha explorado como marcador de la actividad de la enfermedad en la EIII28,29. De manera particular, se ha descrito que la CF se correlaciona estrechamente con la actividad clínica, endoscópica e histológica en la CU, así como con la gravedad y la extensión de la enfermedad ${ }^{30}$. En la EC, la prueba de CF es aún más importante que en la $\mathrm{CU}$, porque con frecuencia la inflamación en la EC suele ser asintomática y puede progresar a complicaciones, y se ha descrito que los valores de CF pueden estar elevados en el $60-70 \%$ de los pacientes con $\mathrm{EC}^{31,32}$. Así mismo, unos valores altos de CF se correlacionan con las puntuaciones principales de evaluación endoscópica. Sin embargo, es importante mencionar que los pacientes que presentan síntomas o signos alarmantes, como sangrado, anemia por deficiencia de hierro, pérdida de peso y fiebre, deben someterse a exploraciones endoscópicas independientemente del valor de la $\mathrm{CF}^{33}$.

\section{La calprotectina fecal en la fase de remisión y como predictor de recaída en la enfermedad inflamatoria intestinal}

La curación de la mucosa se define como la ausencia de friabilidad, ulceración y erosión en la mucosa 
para ambas enfermedades, y ha emergido como un objetivo terapéutico tanto en la práctica clínica como en los ensayos clínicos; sin embargo, requiere repetidas endoscopias y biopsias ${ }^{34}$. Por ello, el uso de la CF se ha asociado con una evaluación predictiva de la Ell con mejores resultados clínicos, como un estado de remisión a largo plazo, evitando endoscopias innecesarias y reduciendo el número de intervenciones quirúrgicas y de hospitalizaciones ${ }^{35,36}$.

La determinación de la CF tiene un papel importante en la predicción de la recaída, así como en el reconocimiento de que el paciente se encuentra en fase de remisión. Se ha encontrado que la CF es una herramienta útil para la toma de decisiones sobre la intensificación o la reducción de la medicación ${ }^{37}$. Por lo tanto, la CF, junto con las características clínicas, puede ayudar a identificar pacientes con riesgo de recaída y a hacer el seguimiento del tratamiento ${ }^{38}$. En la remisión clínica de la CU, las cifras de CF se correlacionan estrechamente con la curación endoscópica de la mucosa, tanto en niños como en adultos. Una concentración baja de CF predice la persistencia de la remisión clínica, en especial en las úlceras asintomáticas de la CU. Se ha demostrado que la CF es un biomarcador útil de cicatrización de la mucosa intestinal ${ }^{39}$.

Un valor de $C F<160 \mathrm{mg} / \mathrm{g}$ se asocia constantemente con enfermedad en fase de remisión, mientras que los valores por encima de ese límite indican una probabilidad de recaída superior al $60 \%$ durante las siguientes 8 semanas ${ }^{40}$.

La CF se ha utilizado en el seguimiento del éxito de la terapia en varios países y ha sido incluida en protocolos de estudios clínicos para evaluar diferentes medicamentos. La terapia con infliximab o adalimumab es un buen predictor de la respuesta clínica a la terapia programada, destacando la inducción del biológico infliximab; en un estudio prospectivo se reportó que el $60 \%$ de los pacientes lograron la remisión endoscópica y los valores de CF disminuyeron a $<50 \mathrm{mg} / \mathrm{kg}$, demostrando así que la concentración de CF es una prueba predictiva de recaída ${ }^{41,42}$. Adicionalmente, en un estudio llevado a cabo por Ferreiro-Iglesias, et al. ${ }^{43}$ en 2016 se halló que los pacientes con Ell en tratamiento de mantenimiento con infliximab tuvieron valores elevados de CF durante 2 meses, lo que permitió predecir la recaída ${ }^{43}$.

En la EC, el biomarcador CF es un sustituto aún más confiable para medir la inflamación intestinal, ya que la EC puede cursar de manera asintomática y progresar a complicaciones, destacando que la CF puede estar elevada en el $60-70 \%$ de los pacientes ${ }^{44}$. Por tanto, una concentración elevada de CF se ha descrito como un biomarcador bien validado de riesgo de recaída. Además, la CF en concentraciones elevadas a pesar de la remisión clínica también se ha detectado en varios estudios tanto en adultos como en niños, mientras que una concentración baja de CF predice la persistencia de la remisión clínica. Así mismo, se ha descrito que los pacientes que requerían colectomía presentaban una concentración de CF significativamente más alta que aquellos que no la necesitaban ${ }^{45,46}$. Finalmente, en las guías clínicas internacionales se recomienda que la prueba de CF sea obligatoria no solo en el diagnóstico, sino también en la evolución de la enfermedad, así como en el seguimiento terapéutico ${ }^{47,48}$. Por lo tanto, la importancia de la determinación de la concentración de la CF contribuye a la evaluación del grado de enfermedad y al seguimiento de la respuesta terapéutica, por lo que es necesario su uso en la Ell.

\section{Financiación}

La presente investigación no ha recibido ninguna beca específica de agencias de los sectores público, comercial o sin ánimo de lucro.

\section{Conflicto de intereses}

Los autores declaran que la investigación se llevó a cabo en ausencia de relaciones comerciales o financieras que pudieran interpretarse como un posible conflicto de intereses.

\section{Responsabilidades éticas}

Protección de personas y animales. Los autores declaran que para esta investigación no se han realizado experimentos en seres humanos ni en animales.

Confidencialidad de los datos. Los autores declaran que han seguido los protocolos de su centro de trabajo sobre la publicación de datos de pacientes.

Derecho a la privacidad y consentimiento informado. Los autores declaran que en este artículo no aparecen datos de pacientes.

\section{Bibliografía}

1. Miyoshi J, Nobutani K, Musch MW, Ringus DL, Hubert NA, Yamamoto M, et al. Time, sex-, and dose-dependent alterations of the gut microbiota by consumption of dietary daikenchuto (TU-100). Evid Based Complement Alternat Med. 2018; 22;2018:7415975. doi: 10.1155/2018/7415975.

2. Seyedian SS, Nokhostin F, Malamir MD. A review of the diagnosis, prevention, and treatment methods of inflammatory bowel disease. J Med Life. 2019;12(2):113-22. doi: 10.25122/jml-2018-0075.

3. Flynn S, Eisenstein S. Inflammatory bowel disease presentation and diagnosis. Surg Clin North Am. 2019;99(6):1051-62. doi: 10.1016/j. suc.2019.08.001. 
4. Di Ruscio M, Vernia F, Ciccone A, Frieri G, Latella G. Surrogate fecal biomarkers in inflammatory bowel disease: rivals or complementary tools of fecal calprotectin? Inflamm Bowel Dis. 2017;24(1):78-92. doi: 10.1093/ ibd/izx011.

5. Huguet JM, Suárez P, Ferrer-Barceló L, Iranzo I, Sempere J. Screening for colorectal cancer in patients with inflammatory bowel disease. Should we already perform chromoendoscopy in all our patients? World J Gastrointest Endosc. 2018;10(11):322-5. doi: 10.4253/wjge.v10.i11.322.

6. Klinger AL, Kann BR. Endoscopy in inflammatory bowel disease. Surg Clin North Am. 2019;99(6):1063-82. doi: 10.1016/j.suc.2019.08.005

7. Kennedy NA, Clark A, Walkden A, Chang JC, Fascí-Spurio F, Muscat M, et al. Clinical utility and diagnostic accuracy of faecal calprotectin for IBD at first presentation to gastroenterology services in adults aged 16-50 years. J Crohns Colitis. 2015;9(1):41-9. doi: 10.1016/j.crohns.2014.07.005

8. Laserna-Mendieta EJ, Lucendo AJ. Faecal calprotectin in inflammatory bowel diseases: a review focused on meta-analyses and routine usage limitations. Clin Chem Lab Med. 2019;57(9):1295-307. doi: 10.1515/cclm-2018-1063.

9. Kerkhoff $C$, Klempt M, Sorg C. Novel insights into structure and function of MRP8 (S100A8) and MRP14 (S100A9). Biochim Biophys. 1998; 1448(2):200-11. doi: 10.1016/s0167-4889(98)00144-x

10. Korndörfer IP, Brueckner F, Skerra A. The crystal structure of the human (S100A8/S100A9)2 heterotetramer, calprotectin, illustrates how conformational changes of interacting alpha-helices can determine specific association of two EF-hand proteins. J Mol Biol. 2007;370(5):887-98. doi: 10.1016/j.jmb.2007.04.065

11. Ayling RM, Kok K. Fecal calprotectin. Adv Clin Chem. 2018;87:161-90. doi: 10.1016/bs.acc.2018.07.005

12. Steinbakk M, Naess-Andresen CF, Lingaas E, Dale I, Brandtzaeg P, Fagerhol MK. Antimicrobial actions of calcium binding leucocyte L1 protein, calprotectin. Lancet. 1990;336(8718):763-5. doi: 10.1016/01406736(90)93237-j.

13. Heida A, Park KT, van Rheenen PF. Clinical utility of fecal calprotectin monitoring in asymptomatic patients with inflammatory bowel disease: a systematic review and practical guide. Inflamm Bowel Dis. 2017;23(6):894902. doi: $10.1097 / \mathrm{MIB} .0000000000001082$.

14. Rokkas T, Portincasa P, Koutroubakis IE. Fecal calprotectin in assessing inflammatory bowel disease endoscopic activity: a diagnostic accuracy meta-analysis. J Gastrointestin Liver Dis. 2018;27(3):299-306. doi: 10.15403/jgld.2014.1121.273.pti.

15. Kanmura S, Hamamoto H, Morinaga $Y$, Oda K, Fujita T, Arima S, et al. Fecal human neutrophil peptide levels correlate with intestinal inflammation in ulcerative colitis. Digestion. 2016;93(4):300-8. doi: 10.1159/000446210.

16. Burri E, Beglinger C. The use of fecal calprotectin as a biomarker in gastrointestinal disease. Expert Rev Gastroenterol Hepatol. 2014;8(2):197-210. doi: 10.1586/17474124.2014.869476.

17. Heilmann RM, Nestler J, Schwarz J, Grützner N, Ambrus A, Seeger J et al. Mucosal expression of S100A12 (calgranulin C) and S100A8/A9 (calprotectin) and correlation with serum and fecal concentrations in dogs with chronic inflammatory enteropathy. Vet Immunol Immunopathol. 2019;211:64-74. doi: 10.1016/j.vetimm.2019.04.003.

18. Mosli MH, Zou G, Garg SK, Feagan SG, MacDonald JK, Chande N, et al. C-reactive protein, fecal calprotectin, and stool lactoferrin for detection of endoscopic activity in symptomatic inflammatory bowel disease patients: a systematic review and meta-analysis. Am J Gastroenterol. 2015;110(6):802-19; quiz 820. doi: 10.1038/ajg.2015.120.

19. Manceau H, Chicha-Cattoir V, Puy H, Peoc'h K. Fecal calprotectin in inflammatory bowel diseases: update and perspectives. Clin Chem Lab Med. 2017;55(4):474-83. doi: 10.1515/cclm-2016-0522.

20. Mumolo MG, Bertani L, Ceccarelli L, Laino G, Di Fluri G, Albano E, et al. Bench to bedside: fecal calprotectin in inflammatory bowel diseases clinical setting World J Gastroenterol. 2018;24(33):3681-94. doi: 10.3748/wjg.v24.i33.3681.

21. Knyazev OV, Kagramanova AV, Korneeva IA, Noskova KK, Belousov SV Parfenov Al. The use of fecal calprotectin in monitoring activity of inflammatory bowel diseases. Ter Arkh. 2019;91(4):53-61. doi: 10.26442/0040 3660.2019.04.000229

22. Caviglia GP, Ribaldone DG, Rosso C, Saracco GM, Astegiano M, Pellicano R. Fecal calprotectin: beyond intestinal organic diseases. Panminerva Med. 2018; 60(1):29-34. doi: 10.23736/S0031-0808.18.03405-5.

23. Lee YW, Lee KM, Lee JM, Chung YY, Kim DB, Kim YJ, et al. The usefulness of fecal calprotectin in assessing inflammatory bowel disease activity. Korean J Intern Med. 2019;34(1):72-80. doi: 10.3904/kjim.2016.324.

24. Simioni J, Skare TL, Campos APB, Kotze L, Messias-Reason I, Ioshii SO, et al. Fecal calprotectin, gut inflammation and spondyloarthritis. Arch Med Res. 2019;50(1):41-6. doi: 10.1016/j.arcmed.2019.04.003.

25. Haisma SM, Galaurchi A, Almahwzi S, Adekanmi Balogun JA, Muller Kobold AC, van Rheenen PF. Head-to-head comparison of three stool calprotectin tests for home use. PLoS One. 2019;14(4):e0214751. doi: 10.1371/journal.pone.0214751.

26. Waugh N, Cummins E, Royle P, Kandala NB, Shyangdan D, Arasaradnam $\mathrm{R}$, et al. Faecal calprotectin testing for differentiating amongst inflammatory and non-inflammatory bowel diseases: systematic review and economic evaluation. Health Technol Assess. 2013;17(55):xv-xix, 1-211. doi: 10.3310/hta17550.
27. Gonczi L, Bessissow T, Lakatos PL. Disease monitoring strategies in inflammatory bowel diseases: what do we mean by "tight control"? World J Gastroenterol. 2019;25(41):6172-89. doi: 10.3748/wjg.v25.i41.6172.

28. Chang S, Malter L, Hudesman D. Disease monitoring in inflammatory bowel disease. World J Gastroenterol. 2015;21(40):11246-59. doi: 10.3748/wjg.v21.i40.11246.

29. Lopez RN, Leach ST, Lemberg DA, Duvoisin G, Gearry RB, Day AS Fecal biomarkers in inflammatory bowel disease. J Gastroenterol Hepatol. 2017;32(3):577-82. doi: 10.1111/jgh.13611.

30. Muthas D, Reznichenko A, Balendran CA, Böttcher G, Clausen IG, Kärrman Mårdh $\mathrm{C}$, et al. Neutrophils in ulcerative colitis: a review of selected biomarkers and their potential therapeutic implications. Scand J Gastroenterol. 2017;52(2):125-35. doi: 10.1080/00365521.2016.1235224.

31. Sipponen T, Kolho KL. Fecal calprotectin in diagnosis and clinical as sessment of inflammatory bowel disease. Scand J Gastroenterol. 2015;50(1):74-80. doi: 10.3109/00365521.2014.987809.

32. Vernia F, Di Ruscio M, Stefanelli G, Viscido A, Frieri G, Latella G. Is fecal calprotectin an accurate marker in the management of Crohn's disease? J Gastroenterol Hepatol. 2020;35:390-400. doi: 10.1111/jgh.14950.

33. Mak WY, Buisson A, Andersen MJ Jr, Lei D, Pekow J, Cohen RD, et al. Fecal calprotectin in assessing endoscopic and histological remission in patients with ulcerative colitis. Dig Dis Sci. 2018;63(5):1294-1301. doi: 10.1007/s10620-018-4980-0.

34. Abraham BP, Ahmed T, Ali T. Inflammatory bowel disease: pathophysiology and current therapeutic approaches. Handb Exp Pharmacol. 2017;239:115-46. doi: 10.1007/164_2016_122.

35. Peyrin-Biroulet L, Reinisch W, Colombel JF, Mantzaris GJ, Kornbluth A, Diamond $\mathrm{R}$, et al. Clinical disease activity, $\mathrm{C}$-reactive protein normalisation and mucosal healing in Crohn's disease in the SONIC trial. Gut. 2014;63(1):88-95. doi: 10.1136/gutjnl-2013-304984.

36. Reinink AR, Lee TC, Higgins PD. Endoscopic mucosal healing predicts favorable clinical outcomes in inflammatory bowel disease: a meta-analysis. Inflamm Bowel Dis. 2016;22(8):1859-69. doi: 10.1097/ MIB.0000000000000816.

37. Carlsen K, Riis LB, Elsberg H, Maagaard L, Thorkilgaard T, Sørbye SW, et al. The sensitivity of fecal calprotectin in predicting deep remission in ulcerative colitis. Scand J Gastroenterol. 2018;53(7):825-30. doi: 10.1080/00365521.2018.1482956

38. Kristensen V, Røseth A, Ahmad T, Skar V, Moum B. Fecal calprotectin: a reliable predictor of mucosal healing after treatment for active ulcerative colitis. Gastroenterol Res Pract. 2017;2017:2098293. doi: 10.1155/2017/2098293.

39. Malvão LDR, Madi K, Esberard BC, de Amorim RF, Silva KDS, Silva KFE, et al. Fecal calprotectin as a noninvasive test to predict deep remission in patients with ulcerative colitis. Medicine (Baltimore). 2021;100(3):e24058. doi: 10.1097/MD.0000000000024058

40. Lin WC, Wong JM, Tung CC, Lin CP, Chou JW, Wang HY, et al. Fecal calprotectin correlated with endoscopic remission for Asian inflammatory bowel disease patients. World J Gastroenterol. 2015;21(48):13566-73. doi: 10.3748/wjg.v21.i48.13566.

41. Tursi A, Elisei W, Picchio M, Giorgetti G, Brandimarte G. Accuracy of rapid fecal calprotectin test in monitoring inflammatory bowel diseases under treatment with TNF $\alpha$ antagonists. Dig Dis Sci. 2015;60(5):1406-13. doi: 10.1007/s10620-014-3459-x.

42. Dreesen E, Baert F, Laharie D, Bossuyt P, Bouhnik $Y$, Buisson A, et al. Monitoring a combination of calprotectin and infliximab identifies patients with mucosal healing of Crohn's disease. Clin Gastroenterol Hepatol. 2020;18(3):637-46.e11. doi: 10.1016/j.cgh.2019.05.029.

43. Ferreiro-Iglesias R, Barreiro-de Acosta M, Otero Santiago M, Lorenzo Gonzalez A, Alonso de la Peña C, Benitez Estevez AJ, et al. Fecal calprotectin as predictor of relapse in patients with inflammatory bowel disease under maintenance infliximab therapy. J Clin Gastroenterol. 2016;50(2):147-51. doi: 10.1097/MCG.0000000000000312.

44. Veauthier B, Hornecker JR. Crohn's disease: diagnosis and management. Am Fam Physician. 2018;98(11):661-9.

45. Jones GR, Fasci-Spurio F, Kennedy NA, Plevris N, Jenkinson P, Lyons M, et al. Faecal calprotectin and magnetic resonance enterography in ileal Crohn's disease: correlations between disease activity and long-term follow-up. J Crohns Colitis. 2019;13(4):442-50. doi: 10.1093/ecco-jcc/ jijy187.

46. Ma C, Battat R, Parker CE, Khanna R, Jairath V, Feagan BG. Update on $C$-reactive protein and fecal calprotectin: are they accurate measures of disease activity in Crohn's disease? Expert Rev Gastroenterol Hepatol. 2019;13(4):319-30. doi: 10.1080/17474124.2019.1563481.

47. Magro F, Gionchetti P, Eliakim R, Ardizzone S, Armuzzi A, Barreiro-de Acosta M, et al. Third European evidence-based consensus on diagnosis and management of ulcerative colitis. Part 1: Definitions, diagnosis, extra-intestinal manifestations, pregnancy, cancer surveillance, surgery, and ileo-anal pouch disorders. J Crohns Colitis. 2017;11(6):649-70. doi: 10.1093/ecco-jcc/jjx008.

48. Lamb CA, Kennedy NA, Raine T, Hendy PA, Smith PJ, Limdi JK, et al. British Society of Gastroenterology consensus guidelines on the management of inflammatory bowel disease in adults. Gut. 2019;68(Suppl 3):s1-s106. doi: 10.1136/gutjnl-2019-318484. 